\title{
Gadobenate Dimeglumine-Enhanced Liver MRI as the Sole Preoperative Imaging Technique: A Prospective Study of Living Liver Donors
}

\section{Su Kyung $A n^{1}$ Jeong Min Lee ${ }^{1}$ Kyung-Suk Suh ${ }^{2}$ Nam Jun Lee ${ }^{2}$ Se Hyung Kim ${ }^{1}$ Young Joon $\mathrm{Kim}^{1}$ Joon Koo Han ${ }^{1}$ Buyng Ihn $\mathrm{Choi}^{1}$}

Keywords: gadobenate dimeglumine, liver disease, liver donor, liver transplantation, MRI

DOI:10.2214/AJR.05.0584

Received April 3, 2005; accepted after revision September 18, 2005.

'Department of Radiology and Institute of Radiation Medicine, Seoul National University College of Medicine, 28, Yongon-dong, Chongno-gu, Seoul 110-744, South Korea. Address correspondence to J. M. Lee (leejm@radcom.snu.ac.kr).

${ }^{2}$ Department of General Surgery, Seoul National University College of Medicine, Seoul, South Korea.

AJR 2006; 187:1223-1233

0361-803X/06/1875-1223

(c) American Roentgen Ray Society

OBJECTIVE. The objective of our study was to determine whether gadobenate dimeglumine-enhanced MRI is practical as the sole preoperative imaging technique for the examination of living liver donors.

SUBJECTS AND METHODS. Forty-four consecutive living donor candidates underwent liver MRI on a 1.5-T MR unit. The MR examination included in- and opposed-phase T1weighted gradient-echo imaging, T2-weighted MR cholangiography, MR angiography (MRA) and parenchymal phase imaging after the administration of gadobenate dimeglumine, and 60minute delayed T1-weighted MR cholangiography. Two abdominal radiologists analyzed the images regarding the depiction of the biliary duct anatomy and the hepatic vascular anatomy and for the presence of focal or diffuse liver disease. The findings were compared with intraoperative cholangiographic and surgical findings in 24 patients who underwent partial hepatectomy.

RESULTS. In the 24 patients who underwent liver harvesting, 10 had biliary anatomic variants confirmed by intraoperative cholangiography. T2-weighted MR cholangiography allowed a correct diagnosis in 75\% $(n=18 / 24)$ and T1-weighted MR cholangiography in 79\% $(n=19 / 24)$ of these patients. When we evaluated the bile duct anatomy using the combined findings of T2and T1-weighted MR cholangiographic images, the diagnostic accuracy increased to $92 \%$ $(n=22 / 24)$, but the difference was not statistically significant $(p>0.05)$. MRA showed a diagnostic accuracy of $79 \%(n=19 / 24)$ for the hepatic arterial anatomy, $100 \%(n=24 / 24)$ for the portal venous anatomy, and $96 \%(n=23 / 24)$ for the hepatic venous anatomy.

CONCLUSION. Gadobenate dimeglumine-enhanced MRI allows comprehensive assessment of the biliary and hepatic vascular systems and the hepatic parenchyma and can serve as the sole preoperative imaging test for living liver donor candidates.

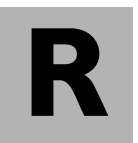
ecent improvements in surgical transplantation techniques have led to a rapid increase in the number of living donor liver transplantations [1]. However, partial hepatectomy carries the risks of various complications including biliary leakage or obstruction and vascular stenosis or bleeding in both donors and recipients [2]. For successful graft acquisition and decreased surgeryrelated complications in living donors, careful preoperative evaluation of the liver anatomy and parenchyma is required.

Complete preoperative imaging of a donor typically includes conventional sonography to evaluate the hepatic steatosis, digital subtraction angiography (DSA) or CT angiography (CTA) to depict the hepatic vascular system, and ERCP or intraoperative cholangiography to assess the biliary anatomy $[3,4]$. This type of imaging workup using several different techniques is time consuming and expensive and is also very tiring for the donors.

Recently, there have been many literature reports indicating that contrast-enhanced MRI has the potential to simplify this complex process $[1,5,6]$. However, existing MR angiography (MRA) and cholangiography techniques have several limitations - that is, their limited temporal and spatial resolution compared with direct DSA and intraoperative cholangiography and defining the optimal contrast agent to obtain the best image quality $[7,8]$.

Based on previous studies, the use of gadobenate dimeglumine for liver MR examinations as a preoperative workup for a living donor transplantation seems to be attractive because of its twofold $\mathrm{T} 1$ relaxation effects and biliary excretion property $[1,9,10]$. The motivation for our study was from the possibility for gadobenate dimeglumine to increase the signal-to-noise ratio (SNR) of 


\section{An et al.}

MRA due to its twofold T1 relaxation effect and its biliary excretion property to be used for 3D cholangiography.

The purpose of this study was to determine the clinical practicability and the diagnostic accuracy of gadobenate dimeglumine-enhanced MRI for the preoperative evaluation of living adult liver donors.

\section{Subjects and Methods Subjects}

At our center, a potential liver donor must be between the ages of 16 and 60 years, be willing to donate, have an identical or compatible blood type and no significant medical or psychologic problems, and have normal findings on liver function tests and negative results for cytotoxic antibody. If the donors have no obvious contraindications to donation in this step, they can undergo radiologic evaluation: sonography, CTA, and T2-weighted MR cholangiography. During a 12-month period (January-December 2004), 44 potential liver donor candidates (15 women, 29 men; age range, 16-58 years; average age, 29 years) were referred for radiologic evaluation. They underwent unenhanced liver MRI, MRA, and T1-weighted MR cholangiography with gadobenate dimeglumine enhancement in addition to the routine radiologic studies mentioned earlier, including sonography, CTA, and T2-weighted MR cholangiography.

All imaging findings were analyzed prospectively in the 44 candidates before the transplantation surgeons assessed surgical candidacy. Finally, 24 candidates underwent partial hepatectomy, and the surgical and intraoperative cholangiographic results were used for comparison with the MRI findings. This study was performed in accordance with institutional review board guidelines, and written informed consent was obtained from all patients.

\section{MR Examination}

We obtained images on a 1.5-T MR scanner (Magnetom Vision, Siemens Medical Solutions) using a maximum gradient strength of $25 \mathrm{mT} / \mathrm{m}$ with a torso phased-array coil. A 220- to 340-cm field of view was used. Before the start of the study, a 22-gauge IV catheter was placed in an antecubital or forearm vein and was attached to an MR-compatible power injector (Spectris, MedRad). Gadobenate dimeglumine (MultiHance, Bracco Imaging) was injected IV at a dose of 0.1 $\mathrm{mmol} / \mathrm{kg}$ of body weight. According to the method detailed by Earls et al. [11], a timing image was then obtained with a test dose of $1 \mathrm{~mL}$ of gadobenate dimeglumine followed by $20 \mathrm{~mL}$ of saline solution, both injected at a rate of $2 \mathrm{~mL} / \mathrm{s}$. This allowed estimation of patient circulation time (bolus transit time from arm vein to abdom- inal aorta) that was used to ensure acquisition of optimal arterial phase images.

\section{MR Sequences and Parameters}

For evaluation of liver parenchyma, breathhold axial T1-weighted in-phase and opposedphase 2D fast low-angle shot (FLASH) imaging (TR, 128 milliseconds; TE for opposed-phase imaging, 2.7 milliseconds; TE for in-phase imaging, 5.3 milliseconds; flip angle, $70^{\circ}$; slice thickness, $7 \mathrm{~mm}$; and matrix, $131 \times 256)$ and T2-weighted HASTE imaging (TR/TE, 4,400/90; flip angle, $150^{\circ}$; slice thickness, $7 \mathrm{~mm}$; and matrix, $131 \times$ 256) were performed.

For T2-weighted MR cholangiography, a multislice HASTE imaging sequence (infinite/95.0; flip angle, $150^{\circ}$; slice thickness, $3 \mathrm{~mm}$ with no interval gap; and matrix, $240 \times 256$ ) and oblique coronal heavily $\mathrm{T} 2$-weighted thick-slab turbo spin-echo (TSE) imaging sequence $(2,800 / 1,100$; flip angle, $150^{\circ} ; 60$-mm-thick slice in coronal oblique planes $\left[-45^{\circ},-25^{\circ},-15^{\circ}, 0^{\circ}, 15^{\circ}, 25^{\circ}, 45^{\circ}\right]$ and 40-mm-thick slice in axial plane; and matrix, $240 \times 256$ ) were performed.

For MRA, a coronal 3D interpolated fat-suppressed spoiled gradient-echo sequence was performed before and after IV administration of gadobenate dimeglumine. The imaging parameters for the sequence were as follows: 4.6/1.8; flip angle, $25^{\circ}$; matrix, $140 \times 256$; field of view, $400-450$ $\mathrm{mm}$; slab thickness, $80 \mathrm{~mm}$ with 40 partitions interpolated to 80 for an effective slice thickness of $1 \mathrm{~mm}$; and an intermittent fat-saturation pulse. The first data set after enhancement, which was timed to show the hepatic arteries using a test dose of gadobenate dimeglumine, was collected an average of 10 seconds after the injection. The second and third data sets for visualization of the portal and hepatic venous systems were collected 60 and 120 seconds after the injection, respectively. For evaluation of incidental focal liver lesions, a second 3D interpolated fat-suppressed spoiled gradient-echo sequence (4.5/1.9; flip angle, $\left.12^{\circ}\right)$ was acquired in the axial plane and included coverage of the entire liver. The acquisition was performed approximately 2-3 minutes after injection. Imaging parameters for the sequence were as follows: matrix, $140 \times 256$; field of view, $320-350 \mathrm{~mm}$; and slab thickness, 160-200 mm with 32-40 partitions interpolated to $64-80$ for an effective slice thickness of $2.5 \mathrm{~mm}$. All acquisition times were kept to less than 25 seconds to facilitate breath-holding at end expiration.

For T1-weighted MR cholangiography, 3D interpolated fat-suppressed spoiled gradient-echo sequences were performed, one in the coronal plane and one in the axial plane, with a time delay of 60 minutes after the administration of gadobenate di- meglumine. This timing was based on our prior experience (unpublished data) because, to our knowledge, there are no documented studies on optimal timing for this agent in this setting. The imaging parameters for the coronal 3D gradient-echo sequence were as follows: $4.5 / 1.9$; flip angle, $12^{\circ}$; matrix, 140 $\times 256$; slab thickness, $81 \mathrm{~mm}$ with 27 partitions interpolated to 54 for an effective slice thickness of 1.5 $\mathrm{mm}$; and an intermittent fat-saturation pulse. The imaging parameters for the axial 3D gradient-echo sequence were identical to those used for equilibrium phase MR images, as described earlier.

\section{Image Processing}

All images were reviewed on a workstation (Virtuoso, Siemens Medical Solutions), a satellite console of the MR unit. One experienced technician performed reformations of the data sets before and after contrast administration. Coronal multiplanar reconstruction images and maximum-intensityprojection (MIP) images for MRA and T2- and T1weighted MR cholangiography were generated interactively from the source images.

\section{Image Interpretation}

All MR images-including source, volume-rendered, and MIP images-were evaluated by consensus of two MR-fellowship-trained abdominal radiologists at a PACS monitor regarding anomalies of the biliary tract; vascular anomalies; focal hepatic masses; diffuse disease of the hepatic parenchyma, such as steatosis; and those diseases that might complicate partial hepatectomy [5]. At the time of this interpretation, the observers had no information regarding correlative findings from other imaging studies. The consensus interpretations performed by the two reviewers of all the MR images were used as the reference standard for the overall assessment of the accuracy of each MR angiographic and MR cholangiographic technique.

Initially, the biliary system was analyzed regarding the anatomic variants. To avoid memory-recollection bias, a minimum of 2 weeks separated the reviews of T2- and T1-weighted MR cholangiography examinations with randomization of the order. In addition to separate analyses of T2- and T1weighted MR cholangiography, a combined analysis of images obtained with the two sequences was performed at a 2-week interval. The observers were unaware of the findings from other sequences.

Normal biliary anatomy was defined when the right posterior duct (from posterior segments VI and VII) drained into the right hepatic duct, and both the right and left hepatic ducts converged into the common hepatic duct. Variants included trifurcation (right posterior duct draining into the junction of the right anterior duct and the left main duct); abnormal right configuration (right posterior 


\section{Preoperative MRI of Living Liver Donors}

duct draining into a left hepatic duct or to the common hepatic duct); accessory hepatic ducts; and other rare variants.

The diagnoses yielded during these three review sessions were later correlated with the intraoperative cholangiographic findings. As the standard reference, intraoperative cholangiography was interpreted by two experienced radiologists who had not participated in the analysis of the MR images. Although we excluded 20 patients who did not undergo liver harvest in analysis of the accuracy due to the lack of a standard of reference, two reviewers determined the findings in accordance between T1- and T2-weighted MR cholangiography in those patients.

The arterial and venous hepatic vascular anatomy was assessed using 3D MRA data sets that included MIP images and source images. Hepatic artery anatomy was classified according to the origins of the right and left hepatic arteries and the presence and the origins of any accessory hepatic arteries [12]. We determined the origin and course of hepatic arteries supplying segment IV. The portal vein anatomy was categorized as normal bifurcation, trifurcation, or abnormal origin of the right anterior portal vein from the left portal vein. Hepatic venous anatomy was evaluated for inferior accessory right hepatic veins and anomalous unions of three hepatic veins $[1,5,6]$.

Evaluation of liver parenchymal abnormalities included assessment of fatty infiltration on the basis of signal loss relative to the spleen on opposedphase T1-weighted gradient-echo images versus inphase images [13, 14]. For quantitative analysis of hepatic steatosis, an abdominal radiologist, with 7 years of experience in abdominal MRI who did not participate in qualitative MR analysis, performed quantitative measurements of signal intensities of the spleen and liver. Region-of-interest (ROI) measurements $\left(100-400 \mathrm{~mm}^{2}\right)$ were made of an area as large as possible to contain only hepatic or splenic parenchyma with no large vessels or biliary trees. At least five ROIs were placed on liver and spleen on five axial slices, and the ROI values were averaged as a mean liver signal intensity. Based on our previous study [15], the percent decrease in the normalized liver signal on the opposed-phase sequence relative to the in-phase sequence was calculated using the following formula and defined as the relative signal decrease:

$$
100 \times\left[L_{i n} / S_{i n}-L_{o p} / S_{o p}\right] / L_{i n} / S_{i n},
$$

where $L_{\text {in }}$ and $S_{\text {in }}$ represent signal for liver and spleen on in-phase images, respectively, and $L_{o p}$ and $S_{o p}$ represent signal for liver and spleen on opposed-phase images. Based on a previous study [15], the cutoff value of relative signal decrease in the liver indicating an inappropriate level of hepatic steatosis was 20 .

The detection and characterization of masses relied on T1- and T2-weighted imaging and dynamic contrast-enhanced T1-weighted imaging. The results of MRI regarding fatty infiltration and focal liver lesions were compared with pathologic results of biopsy from the transplanted liver and the results of combined interpretation of sonography and CT by two radiologists who had not participated in the analysis of the MR images.

\section{Intraoperative Correlation}

Twenty-four subjects underwent laparotomy and had successful right hepatic lobectomy for donation to liver transplant recipients. Intraoperative findings were compared with MRI findings in all 24 patients who underwent intraoperative cholangiography. As part of the surgical procedure, the hepatic artery and portal vein were dissected only in the hepatic hilum, and before the operation, the transplantation surgeons were informed of the results of CTA regarding vascular variants. Intraoperative cholangiography was used as the reference standard examination for evaluation of biliary variants. In the cases in which the intraoperative cholangiographic and MR cholangiographic findings were discordant, the MR and intraoperative images were compared side by side to assess the causes of the discrepant findings.

\section{Statistical Analysis}

To compare the diagnostic performances, including sensitivity, specificity, and overall accuracy, of the two individual MR cholangiographic techniques and a combination of T1- and T2weighted MR cholangiographic images with that of intraoperative cholangiography, the McNemar test was used (SPSS software [version 11.0], Statistical Package for the Social Sciences). A $p$ value of less than 0.05 was considered to indicate statistical significance.

\section{Results}

Of the 44 subjects who were included in our study, 20 (45\%) were excluded as transplantation candidates because of the following reasons. In two patients, macrovesicular steatosis of more than $60 \%$ was confirmed at biopsy; at our institution, donors with greater than $30 \%$ histologically determined macrovesicular steatosis are generally rejected. Other reasons for exclusion of the donor candidates were a relatively small volume of liver for transplantation in one, incidentally detected stomach cancer in one, asthma in one, diabetes mellitus in one, change of recipient to another donor candidate in one or to a cadaver in three, death of recipi- ent in five, resignation of donation in four, and transfer to other hospital in one patient. None of the patients was excluded from being a transplantation candidate due to biliary anomalies. Finally, 24 candidates (eight women, 16 men; age range, 20-58 years; average age, 28 years) ultimately underwent partial hepatectomy (right hepatectomy in 17 patients, left hepatectomy in three patients, left lateral segmentectomy in four patients) with intraoperative cholangiography.

None of the donors who underwent partial hepatectomy experienced biliary ductal or vascular complications related to harvesting. The results of imaging and operative findings are described next according to their anatomic divisions-namely, biliary, vascular, and parenchymal findings. The quality of the MR images was satisfactory to interpret these findings except in one patient with somewhat early venous phase MRA. The results from surgery are summarized in Table 1.

\section{Biliary Ductal System}

In the 24 patients who underwent liver harvesting, 10 had biliary anatomic variants that were confirmed by intraoperative cholangiography and surgical findings (Figs. 1 and 2). MRI findings and intraoperative cholangiographic findings are summarized in Table 2.

Compared with interpretation of the intraoperative cholangiographic images as the reference standard, T2-weighted MR cholangiography correctly depicted the biliary anatomy in $18(75 \%)$ of the 24 subjects, including 12 of 14 with normal biliary anatomy and six of 10 with biliary anatomy variants. These results represented an overall sensitivity of $60 \%$ and an overall specificity of $86 \%$ for the detection of biliary anatomy variants.

When considered separately, gadobenate dimeglumine-enhanced T1-weighted MR cholangiography correctly depicted the biliary anatomy in 19 (79\%) of the 24 subjects, including all 14 of the subjects with normal anatomy and five of 10 with variants, for a sensitivity of $50 \%$ and a specificity of $100 \%$ for the detection of variants. In contrast, combined interpretation of both MR cholangiographic image sets resulted in an accurate definition of the biliary anatomy in $22(92 \%)$ of the 24 candidates, including 13 of the 14 donors with normal biliary anatomy and nine of the 10 donors with variants, for a sensitivity of $90 \%$ and a specificity of $93 \%$ for the detection of variants. Gadobenate dimeglumine-enhanced T1-weighted MR cholangiography was more accurate than 


\section{An et al.}

TABLE I: Vascular and Biliary Anatomy in 24 Patients Who Underwent Partial Hepatectomy

\begin{tabular}{|c|c|c|c|c|c|c|c|c|}
\hline \multicolumn{3}{|c|}{ Patient } & \multirow[b]{2}{*}{ Graft } & \multirow[b]{2}{*}{ Bile Duct } & \multirow[b]{2}{*}{ Hepatic Artery } & \multirow[b]{2}{*}{ Portal Vein } & \multirow[b]{2}{*}{ Hepatic Vein } & \multirow[b]{2}{*}{ Parenchyma } \\
\hline No. & Sex & Age (y) & & & & & & \\
\hline 1 & $\mathrm{M}$ & 23 & Right hemihepatectomy & $\begin{array}{l}\text { Trifurcation of RAD, RPD, } \\
\text { and LHD }\end{array}$ & & & & \\
\hline 2 & $\mathrm{M}$ & 24 & Right hemihepatectomy & RPD draining to LHD & & & & \\
\hline 3 & $\mathrm{~F}$ & 35 & $\begin{array}{l}\text { Left lateral } \\
\text { segmentectomy }\end{array}$ & & Replaced LHA from LGA & & & \\
\hline 4 & $\mathrm{M}$ & 25 & Right hemihepatectomy & & & & RIHV & \\
\hline 5 & $\mathrm{M}$ & 25 & $\begin{array}{l}\text { Left lateral } \\
\text { segmentectomy }\end{array}$ & $\begin{array}{l}\text { Segment VII bile duct } \\
\text { draining to LHD }\end{array}$ & $\begin{array}{l}\text { Early branching segment } \\
\text { IV artery }\end{array}$ & & & \\
\hline 6 & $\mathrm{~F}$ & 58 & Left hemihepatectomy & & & & & \\
\hline 7 & M & 27 & $\begin{array}{l}\text { Modified right } \\
\text { hemihepatectomy }\end{array}$ & & & $\begin{array}{l}\text { Common cloaca of } \\
\text { right PV }\end{array}$ & RIHV & \\
\hline 8 & $\mathrm{M}$ & 24 & Right hemihepatectomy & & Replaced LHA from LGA & & & \\
\hline 9 & M & 24 & Right hemihepatectomy & RPD draining to LHD & Accessory RHA from SMA & & & \\
\hline 10 & M & 29 & Left hemihepatectomy & RPD draining to LHD & & & & \\
\hline 11 & $\mathrm{~F}$ & 25 & $\begin{array}{l}\text { Left lateral } \\
\text { segmentectomy }\end{array}$ & & $\begin{array}{l}\text { Accessory LHA from LGA; } \\
\text { replaced PHA from SMA }\end{array}$ & $\begin{array}{l}\text { Right anterior PV } \\
\text { arising from left PV }\end{array}$ & RIHV & \\
\hline 12 & $\mathrm{~F}$ & 43 & Right hemihepatectomy & & & & RIHV & \\
\hline 13 & M & 20 & Right hemihepatectomy & $\begin{array}{l}\text { Segment } V \text { bile duct } \\
\text { draining to CHD }\end{array}$ & & & & \\
\hline 14 & $\mathrm{~F}$ & 34 & $\begin{array}{l}\text { Left lateral } \\
\text { segmentectomy }\end{array}$ & $\begin{array}{l}\text { RPD draining to LHD; } \\
\text { bile duct hypoplasia }\end{array}$ & Accessory LHA from LGA & & & \\
\hline 15 & $\mathrm{M}$ & 20 & Right hemihepatectomy & RAD draining to CHD & & Trifurcation & & \\
\hline 16 & $\mathrm{M}$ & 22 & Right hemihepatectomy & & & Trifurcation & RIHV & \\
\hline 17 & $\mathrm{M}$ & 31 & Right hemihepatectomy & & $\begin{array}{l}\text { Early branching segment } \mathrm{V} \\
\text { artery }\end{array}$ & & RIHV & \\
\hline 18 & $\mathrm{M}$ & 24 & Right hemihepatectomy & & $\begin{array}{l}\text { Accessory LHA from LGA; } \\
\text { early branching segment } V \\
\text { artery }\end{array}$ & & RIHV, RMHV & \\
\hline 19 & $\mathrm{~F}$ & 36 & Right hemihepatectomy & & & & SHV & \\
\hline 20 & $\mathrm{M}$ & 22 & Right hemihepatectomy & & & & & \\
\hline 21 & $\mathrm{M}$ & 23 & Right hemihepatectomy & $\begin{array}{l}\text { Segment VI bile duct } \\
\text { draining to CHD }\end{array}$ & & & & \\
\hline 22 & $\mathrm{M}$ & 30 & Left hemihepatectomy & $\begin{array}{l}\text { Bifurcation of segment II } \\
\text { and segment III bile duct }\end{array}$ & & Trifurcation & & \\
\hline 23 & $\mathrm{~F}$ & 22 & Right hemihepatectomy & & Replaced LHA from LGA & & & \\
\hline 24 & $\mathrm{~F}$ & 33 & Right hemihepatectomy & & $\begin{array}{l}\text { Accessory LHA from LGA; } \\
\text { replaced RHA from SMA }\end{array}$ & & RIHV & $\begin{array}{l}\text { Hemangioma } \\
\text { in segment } \\
\text { VI }\end{array}$ \\
\hline
\end{tabular}

Note- $\mathrm{RAD}=$ right anterior duct, $\mathrm{RPD}=$ right posterior duct, $\mathrm{LHD}=$ left hepatic duct, $\mathrm{LHA}=$ left hepatic artery, $\mathrm{LGA}=$ left gastric artery, RIHV = right inferior accessory hepatic vein, $\mathrm{PV}=$ portal vein, $\mathrm{RHA}=$ right hepatic artery, $\mathrm{SMA}=$ superior mesenteric artery, $\mathrm{PHA}=$ proper hepatic artery, $\mathrm{CHD}=$ common hepatic duct, $\mathrm{RMHV}=$ right middle accessory hepatic vein, SHV = superior accessory hepatic vein.

T2-weighted MR cholangiography for depicting biliary anatomy, although differences did not reach statistical significance $(p=1.00)$. However, when we evaluated the bile duct anatomy by combined interpretations of the T2- and T1-weighted MR cholangiographic images, the diagnostic accuracy increased to 92\%. However, the McNemar test failed to show a statistically significant increase compared with each T1-weighted $(p=0.248)$ and
T2-weighted ( $p=0.134)$ MR cholangiographic technique.

The differences in sensitivity and specificity among the three interpretation methods for determining the presence of biliary anatomic variants were not statistically significant according to the McNemar test ( $p=0.13$ for T1-weighted versus combined T1- and T2-weighted MR cholangiography, $p=0.25$ for $\mathrm{T} 2$-weighted versus combined
T1- and T2-weighted MR cholangiography). In the 24 patients who underwent liver harvest, the accordance between T1- and T2weighted MR cholangiography was $79 \%$ ( $n=19 / 24)$. In addition, in the 20 patients who did not undergo liver harvest and were excluded from analysis of the accuracy of MR cholangiography, T1- and T2-weighted MR cholangiography showed concordant results in 15 patients $(75 \%)$. 


\section{Preoperative MRI of Living Liver Donors}
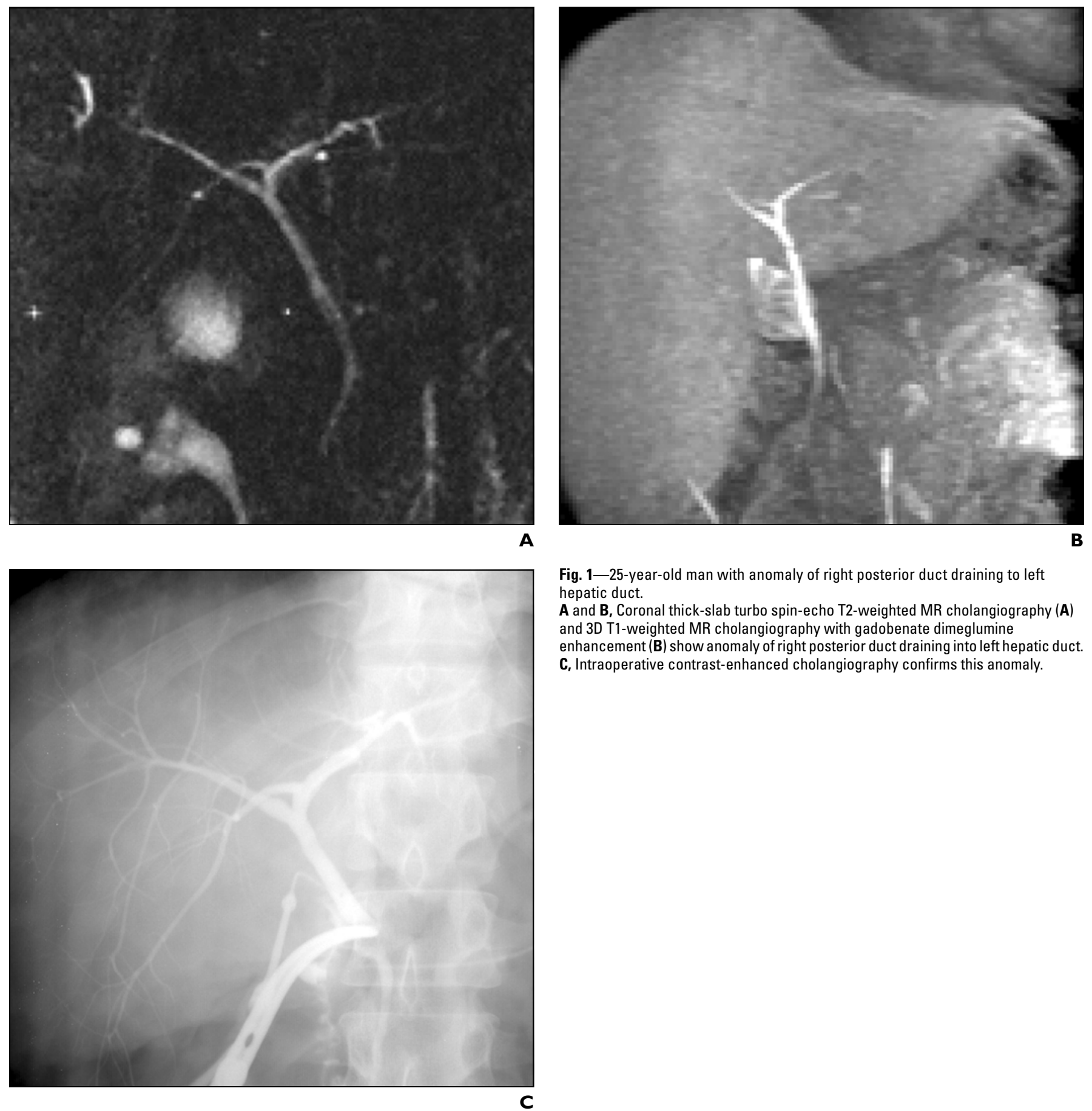

Fig. 1-25-year-old man with anomaly of right posterior duct draining to left hepatic duct.

A and B, Coronal thick-slab turbo spin-echo T2-weighted MR cholangiography (A) and $3 \mathrm{D}$ T1-weighted MR cholangiography with gadobenate dimeglumine

enhancement (B) show anomaly of right posterior duct draining into left hepatic duct

C, Intraoperative contrast-enhanced cholangiography confirms this anomaly.

\section{Vascular System}

More than half of our patients ( $n=14 / 24$, $58 \%$ ) were found to have normal hepatic artery anatomy. Thirteen arterial anatomic variants were observed in 10 patients from surgery: an accessory left hepatic artery originating from the left gastric artery
( $n=4,31 \%)$; a left hepatic artery originating from the left gastric artery $(n=3,23 \%)$; a right hepatic artery from the superior mesenteric artery $(n=1,8 \%)$ (Fig. 3 ); an accessory right hepatic artery from the superior mesenteric artery $(n=1,8 \%)$; a proper hepatic artery from the superior mesenteric ar- tery $(n=1,8 \%)$; and early branching segmental arteries $(n=3,23 \%)$. The artery to segment IV arose from the left hepatic artery in 21 donors and from the right hepatic artery in three. In these three donors, the distance from the hepatic artery bifurcation to the origin of the artery supplying segment IV 


\section{An et al.}
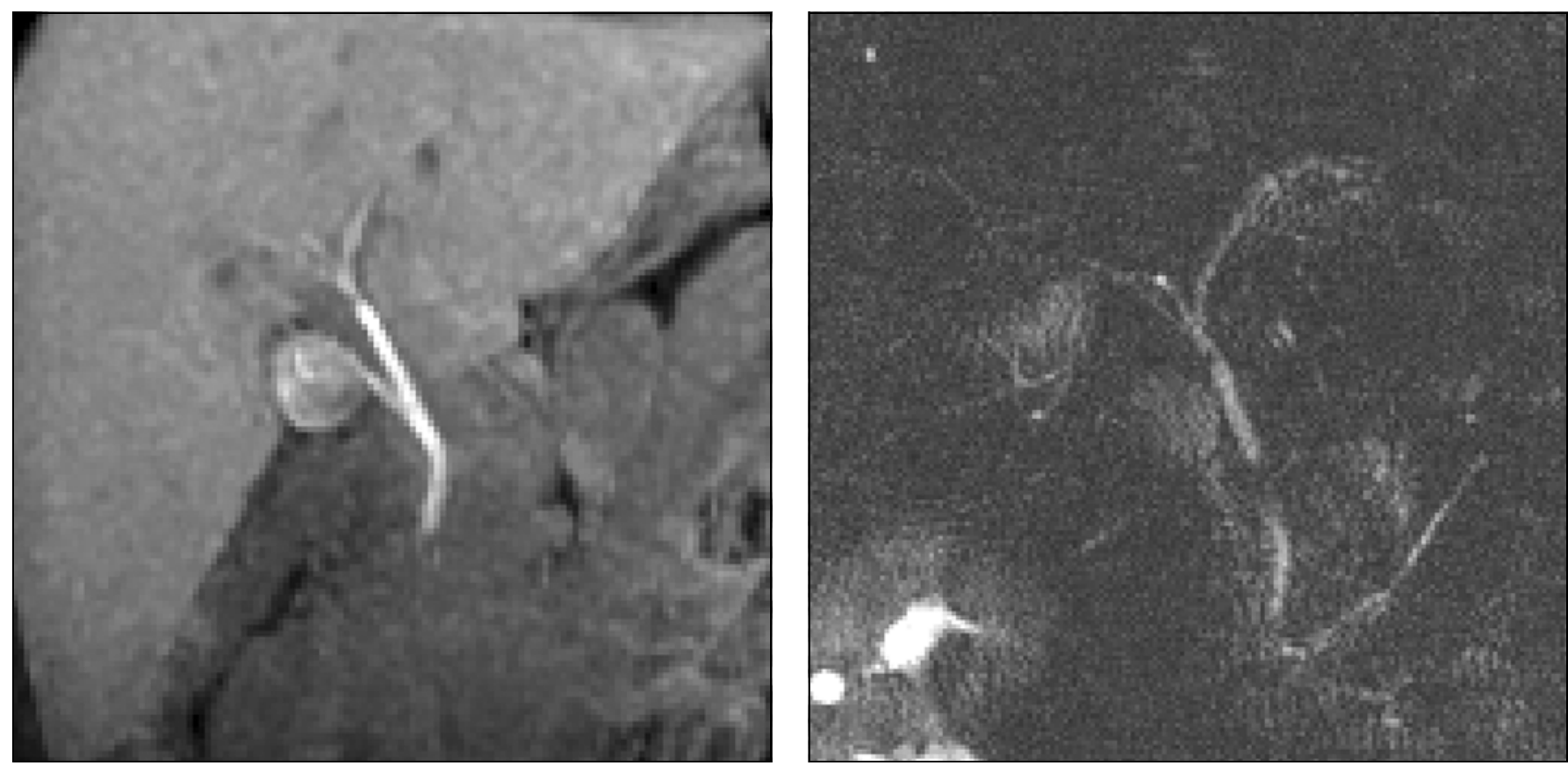

A

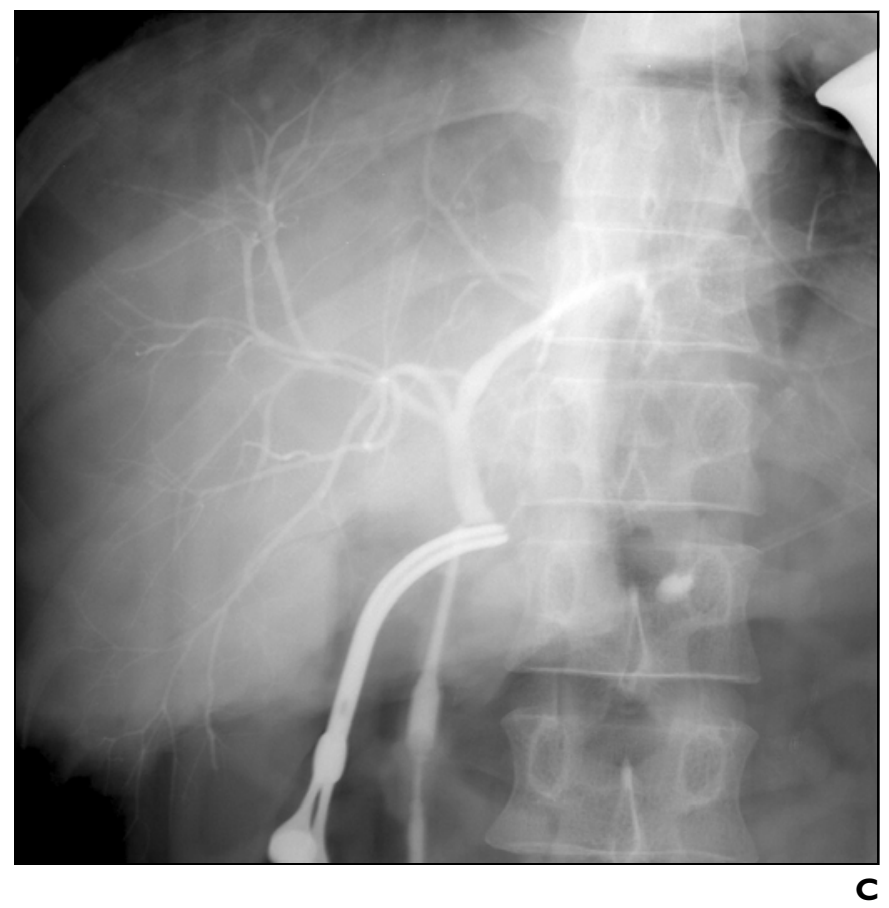

Fig. 2-23-year-old man with biliary trifurcation.

A and B, Coronal thick-slab T2-weighted MR cholangiography (A) and 3D T1weighted MR cholangiography with gadobenate dimeglumine enhancement (B) show anomaly of trifurcation of bile duct at conjugation level.

C, Intraoperative contrast-enhanced cholangiography confirms this anomaly.

was less than $1.5 \mathrm{~cm}$. MRA revealed the origin of the artery to segment IV correctly in all cases. However, MRA did not reveal two accessory left hepatic arteries and three early branching segmental arteries. Therefore, the diagnostic accuracy of MRA was $79 \%$ $(n=19 / 24)$.
Portal venous variants were confirmed in $21 \%$ of our patients $(n=5 / 24)$ during surgery: trifurcation of the right anterior portal vein, the right posterior portal vein, and the left portal vein in three patients (Fig. 4); common cloaca of right portal vein in one patient; and a right anterior portal vein originating from the left portal vein in one patient. In all patients $(n=24 / 24)$, MRA in the portal venous phase produced the correct diagnosis.

Hepatic accessory veins greater than $5 \mathrm{~mm}$ in diameter were identified in $38 \%$ of our patients $(n=9 / 24)$ during surgery (Fig. 5). Of these patients with a right accessory hepatic 
Preoperative MRI of Living Liver Donors

TABLE 2: MR Findings and Intraoperative Cholangiographic Findings in 24 Patients Who Underwent Partial Hepatectomy

\begin{tabular}{|c|c|c|c|c|}
\hline $\begin{array}{l}\text { Patient } \\
\text { No. }\end{array}$ & T2-Weighted Images Only & T1-Weighted Images Only & $\begin{array}{l}\text { Combined Interpretation } \\
\text { of T2- and T1-Weighted Images }\end{array}$ & Intraoperative Cholangiography \\
\hline 2 & RPD draining to LHD & RPD draining to LHD & RPD draining to LHD & RPD draining to LHD \\
\hline 3 & & & & \\
\hline 4 & & & & \\
\hline 5 & Trifurcation of RAD, RPD, and LHD & RPD draining to LHD & RPD draining to LHD & Segment VII bile duct draining to LHD \\
\hline 7 & & & & \\
\hline 8 & & & & \\
\hline 9 & RPD draining to LHD & RPD draining to LHD & RPD draining to LHD & RPD draining to LHD \\
\hline 10 & RPD draining to LHD & RPD draining to LHD & RPD draining to LHD & RPD draining to LHD \\
\hline 11 & & & & \\
\hline 15 & RAD draining to $\mathrm{CHD}$ & & RAD draining to CHD & RAD draining to $\mathrm{CHD}$ \\
\hline 16 & & & & \\
\hline 17 & & & & \\
\hline 18 & & & & \\
\hline 19 & Accessory segment III bile duct & & & \\
\hline 20 & Trifurcation of RAD, RPD, and LHD & & Trifurcation of RAD, RPD, and LHD & \\
\hline 21 & $\begin{array}{l}\text { Segment VI bile duct draining to } \\
\text { CHD }\end{array}$ & & $\begin{array}{l}\text { Segment VI bile duct draining to } \\
\text { CHD }\end{array}$ & Segment VI bile duct draining to CHD \\
\hline 22 & & & $\begin{array}{l}\text { Bifurcation of segment II and } \\
\text { segment III bile duct }\end{array}$ & $\begin{array}{l}\text { Bifurcation of segment II and segment } \\
\text { III bile duct }\end{array}$ \\
\hline
\end{tabular}

vein, eight underwent right hepatectomy during which the vessel was successfully reimplanted in the transplant recipient. We missed the vessel in one patient because the quality of the hepatic venous phase MRA images was inadequate. Therefore, the diagnostic accuracy of MRA was $96 \%(n=23 / 24)$.

\section{Hepatic Parenchyma}

Of the 44 patients who underwent gadobenate dimeglumine-enhanced MRI before donation, we diagnosed fatty liver in seven $(16 \%)$ by relative signal decrease measured from liver signal intensity on in- and opposed-phase MR images (> 20 images). Two patients were eliminated because of advanced hepatic steatosis seen on in- and opposed-phase T1-weighted gradient-echo images and confirmed by biopsy (macroste- atosis of more than 60\%) (Fig. 6). Their relative signal decreases were 62 and 66, respectively. Three other patients with fatty liver (relative signal decrease: 23, 27, 22) were eliminated for other reasons and did not undergo biopsy. The remaining two patients (relative signal decrease: 31,28 ) underwent harvesting after fatty liver improvement was proven by repeated biopsy and the normal range of macrosteatosis was proven by pathologic results of biopsy from the transplanted liver.

The remaining 22 patients who showed no evidence of advanced hepatic steatosis on in- and opposed-phase images (mean relative signal decrease $=1.3 \pm 14$ ) underwent harvesting and were confirmed to have no steatosis or minimal steatosis $(<5 \%)$ by biopsy from transplanted liver. Two patients had developed hemangioma, as shown on MR images and confirmed by dynamic CT; however, this finding did not affect the decision about whether to harvest. T1-weighted MR cholangiography revealed numerous peribiliary cysts in one patient; that patient underwent successful harvesting, and peribiliary cysts were confirmed by biopsy. However, CT and T2-weighted MR cholangiography could not be used to differentiate peribiliary cysts from choledochal cysts in that patient (Fig. 7).

\section{Discussion}

Recently, MRI has proven to be adequate for providing the parenchymal, ductal, and vascular information required to optimize the harvest procedure of living liver donors. By replacing CT with MRI for donor assessment, we can 


\section{An et al.}

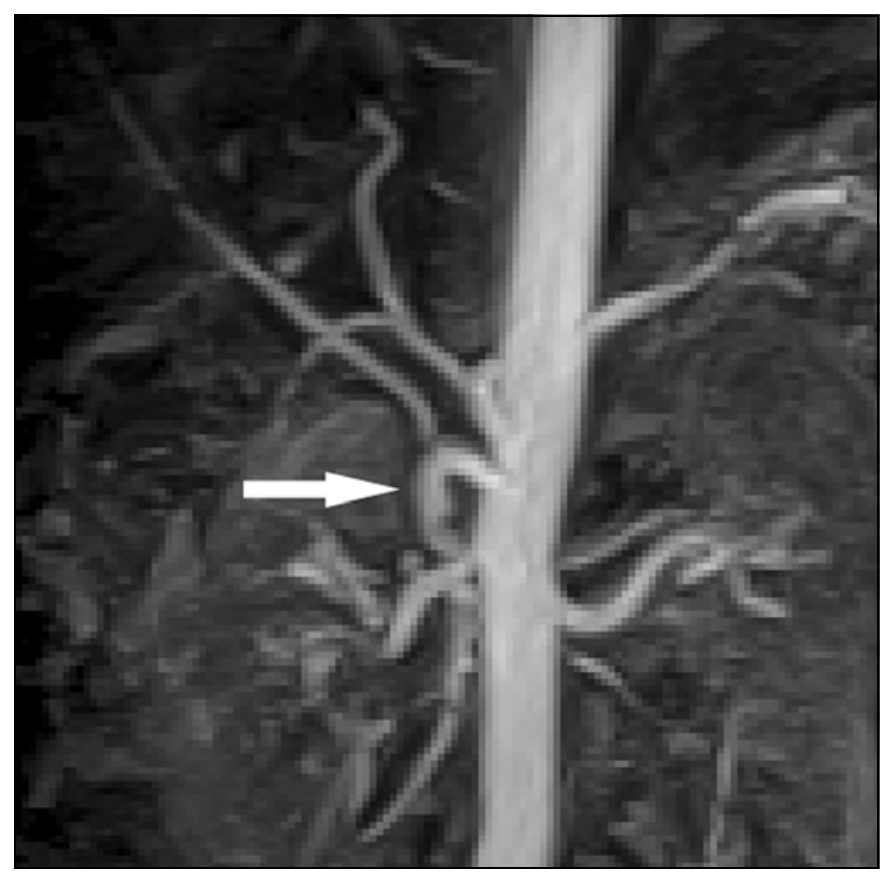

Fig. 3-33-year-old woman with replaced right hepatic artery. Coronal maximumintensity-projection image obtained from arterial phase 3D MR angiography with gadobenate dimeglumine enhancement shows anomaly of right hepatic artery arising from superior mesenteric artery (arrow).

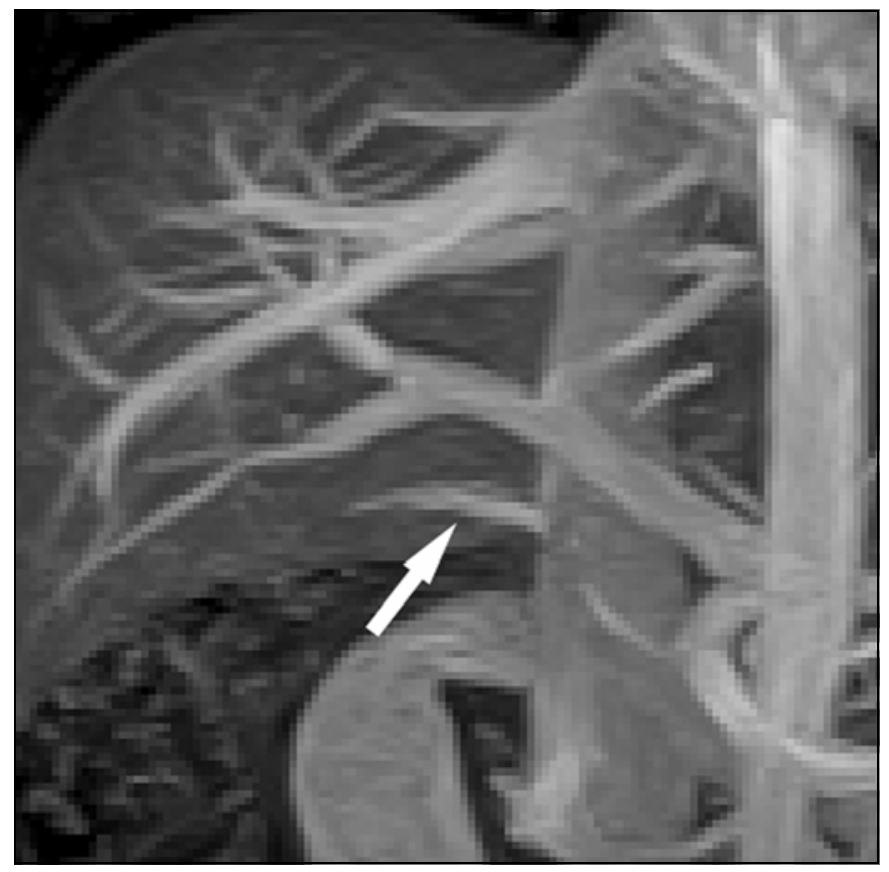

Fig. 5-43-year-old woman with right inferior accessory hepatic vein. Coronal maximum-intensity-projection image obtained from hepatic venous phase 3D MR angiography with gadobenate dimeglumine enhancement shows anomaly of right inferior accessory hepatic vein (arrow) draining directly into inferior vena cava.

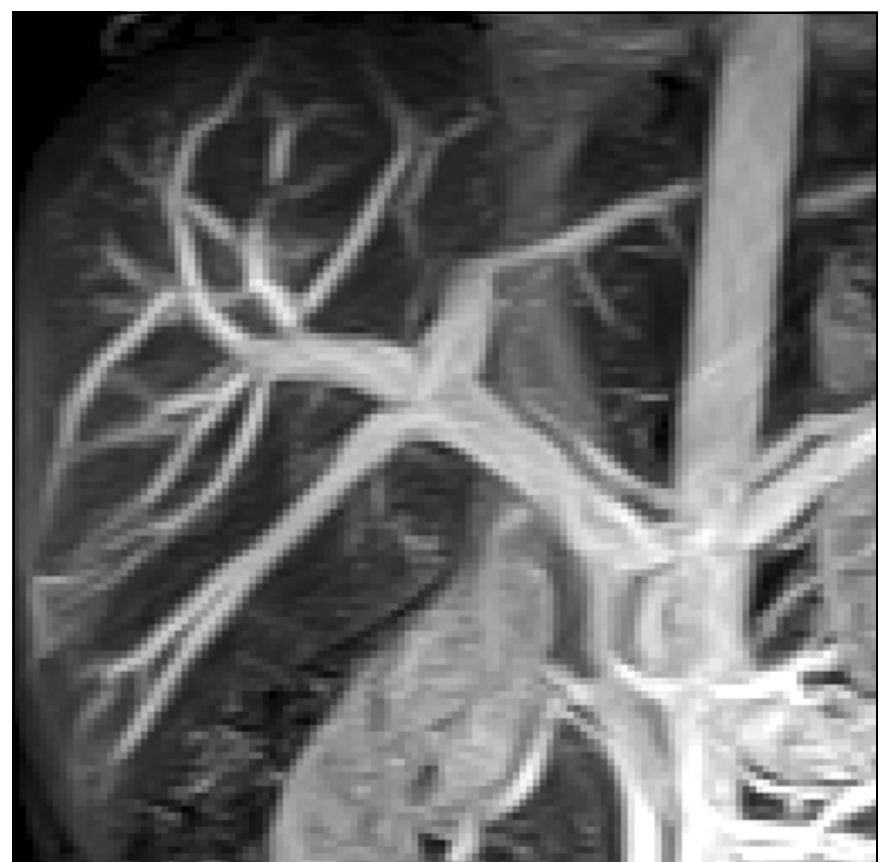

Fig. 4-22-year-old man with portal vein trifurcation. Coronal maximum-intensityprojection image obtained from portal venous phase 3D MR angiography with gadobenate dimeglumine enhancement shows anomaly of trifurcation of right anterior portal vein, right posterior portal vein, and left portal vein.

avoid exposing the potential donor to ionizing radiation and nephrotoxic contrast agents. Furthermore, the MR-based strategy eliminates the need for invasive catheter angiography and ERCP, thus considerably reducing the cost of the workup and the associated pain and potential risk of complications $[1,5,6]$.

However, MRA and cholangiography have limited temporal and spatial resolution compared with direct DSA and intraoperative cholangiography. From this point of view, we speculate that the twofold T1 relaxation afforded by gadobenate dimeglumine provides a higher SNR of the vascular structure on MRA than other gadolinium chelates and its biliary excreting feature contributes to improving the evaluation of the biliary anatomy by allowing delayed 3D T1-weighted MR cholangiography $[9,10]$.

Indeed, in our study, combined T2- and T1-weighted MR cholangiography increased the diagnostic accuracy to $92 \%$ from $75 \%$ on T2and from $79 \%$ on T1-weighted MR cholangiography. Many previous studies have shown that MR cholangiography, using either the T2weighted TSE or HASTE sequence, clearly shows the biliary anatomy, but some biliary anomalies are not easily revealed because of the limited resolution and 2D character of T2-weighted MR cholangiography [16-18]. In addition, given that the complex orthogonal relationships between the right anterior duct, right posterior duct, left hepatic duct, and common hepatic duct are often difficult to define with confidence on conventional 2D MR images, the use of volume-rendering algorithms for reconstruction of gadobenate dimeglumine-enhanced 3D data sets facilitates definition of these relationships for surgical planning [19]. Furthermore, gadobenate dimeglumine-enhanced T1-weighted MR cholangiography can provide information about the differentiation of 
cystic structures near bile ducts and bile duct lumen. For example, in one patient with numerous peribiliary cysts, no communication of the cysts with a bile duct was confirmed on delayed T1-weighted contrast-enhanced MR cholangiography. Moreover, CT and normal T2-weighted MR cholangiography could not be used to differentiate peribiliary cysts from choledochal cysts.

Recently, several studies using mangafodipir trisodium-enhanced 3D T1-weighted MR cholangiography have reported increased SNR and a greater depiction rate of the ductal anatomy than T2-weighted MR cholangiography and 2D gradient-echo images [7, 19, 20]. In our study, T1-weighted 3D gradientecho images that were obtained 1 hour after injection of gadobenate dimeglumine revealed contrast excretion into the biliary tree and provided cholangiographic images. However, given that only $2-5 \%$ of injected gadobenate dimeglumine was excreted into a bile duct but approximately $50 \%$ of mangafodipir trisodium was excreted into a bile duct, the SNR of bile on mangafodipir trisodium-enhanced cholangiography could be much higher than that on gadobenate dimeglumine-enhanced cholangiography. Nevertheless, gadobenate dimeglumine-enhanced MRI could have potential advantages over mangafodipir trisodium-enhanced imaging because of its utility for dynamic first-pass imaging. Indeed, given that preoperative evaluation of liver donor candidates includes precise anatomic information about hepatic vasculature, parenchymal abnormalities, and biliary anomalies, the capability of gadobenate dimeglumine to allow simultaneous acquisition of T1-weighted MR cholangiography and parenchymal and vascular imaging has additional value over mangafodipir trisodium. In addition, gadobenate dimeglumine is easier of use than mangafodipir trisodium, which needs to be infused slowly over 10-20 minutes, and is less expensive than mangafodipir trisodium.

Several recent reports show CT cholangiography with iodipamide meglumine enables good visualization of biliary tract anatomy [21, 22]. Wang et al. [21] reported that biliary tract anatomy depicted on CT cholangiography was concordant with findings at surgery in $96 \%$ of 24 subjects who underwent right lobe retrieval. However, despite the fact that IV cholangiography with iodipamide meglumine has high accuracy in biliary tract imaging, it also has some risks of contrast material reactions and additional iodinated contrast agent needs to be administered to provide adequate hepatic vascular angiography and parenchymal imaging. In our institute where more than 200 living liver donor transplantations have been performed without a donor death to date, we always give the first priority to the safety of the liver donor. Furthermore, our result in diagnostic accuracy of combined T2- and T1-weighted MR cholangiography $(92 \%)$ is comparable with the results of Wang et al. Therefore, considering that gadobenate dimeglumine is safer than iodipamide meglumine, use of additional contrast agent for vascular and parenchymal evaluation is not needed, and diagnostic accuracy is comparable, a combined approach of T2-weighted MR cholangiography and gadobenate dimeglumine-enhanced MRA and MR cholangiography could have reasonable standing as a valuable diagnostic imaging tool for liver donor evaluation.

Our results showed an accuracy of $79 \%$ for diagnosis of hepatic arterial anatomy, $100 \%$ for portal venous anatomy, and $96 \%$ for hepatic venous anatomy. Although the spatial resolution of MRI for the evaluation of hepatic arterial anatomy remains inferior to that of DSA, MRA has, in our experience, proven to be an accurate preoperative test for the definition of the portal and hepatic venous anatomy. Several studies have found that CT or MRI can show the anatomy of the hepatic vasculature of a liver donor before transplantation [23-25]. Winter et al. [23] and Chan et al. [24] showed that in liver transplantation candidates, successful 3D CT arteriography was as accurate as catheter angiography for assessing hepatic arterial anatomy. Although the risk of radiation exposure and of a reaction to iodinated contrast material is probably low enough to justify the use of CT for the evaluation of the hepatic vasculature of a living liver donor, the superior safety profile of gadolinium-based agents compared with that of the iodinated contrast agents must be considered one merit of MRI [26, 27]. In addition, when measured using a test dose of contrast material, arterial phase 3D MR images can be obtained reliably and reproducibly [11].
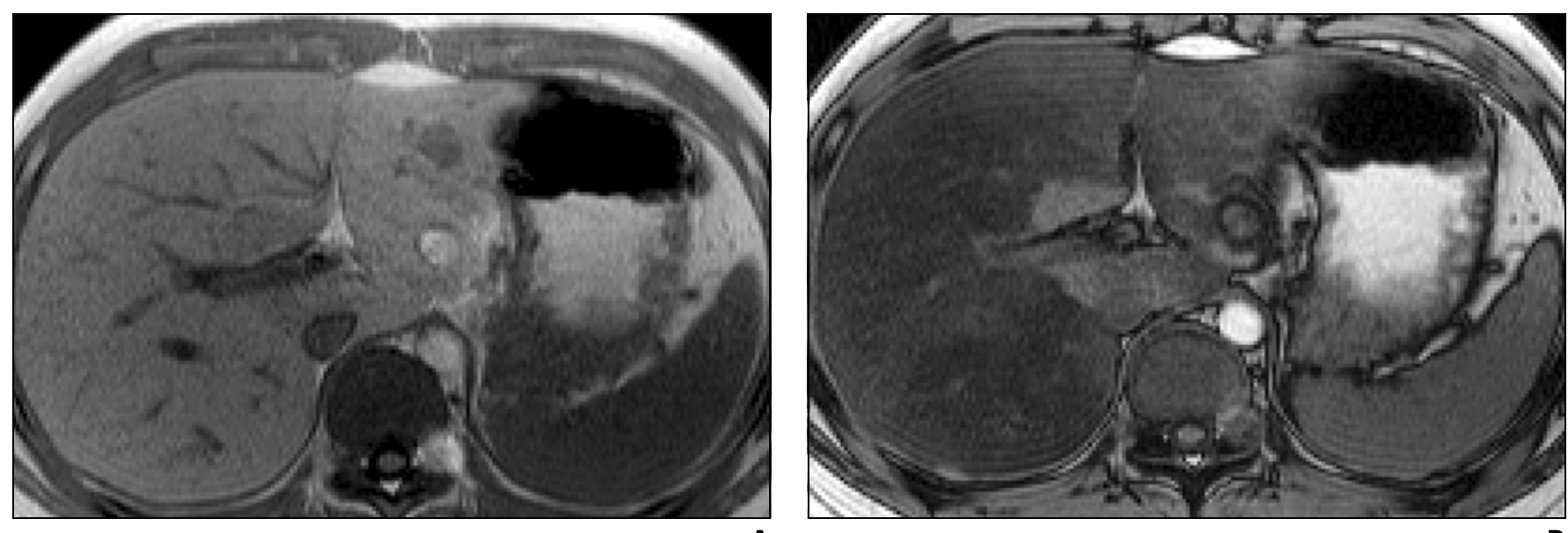

A

Fig. 6-16-year-old boy with uneven fatty infiltration of liver.

A and B, In-phase (A) and opposed-phase (B) T1-weighted gradient-echo images show signal drop in liver, suggesting fatty liver with focal sparing in segment IV and segment I. 


\section{An et al.}
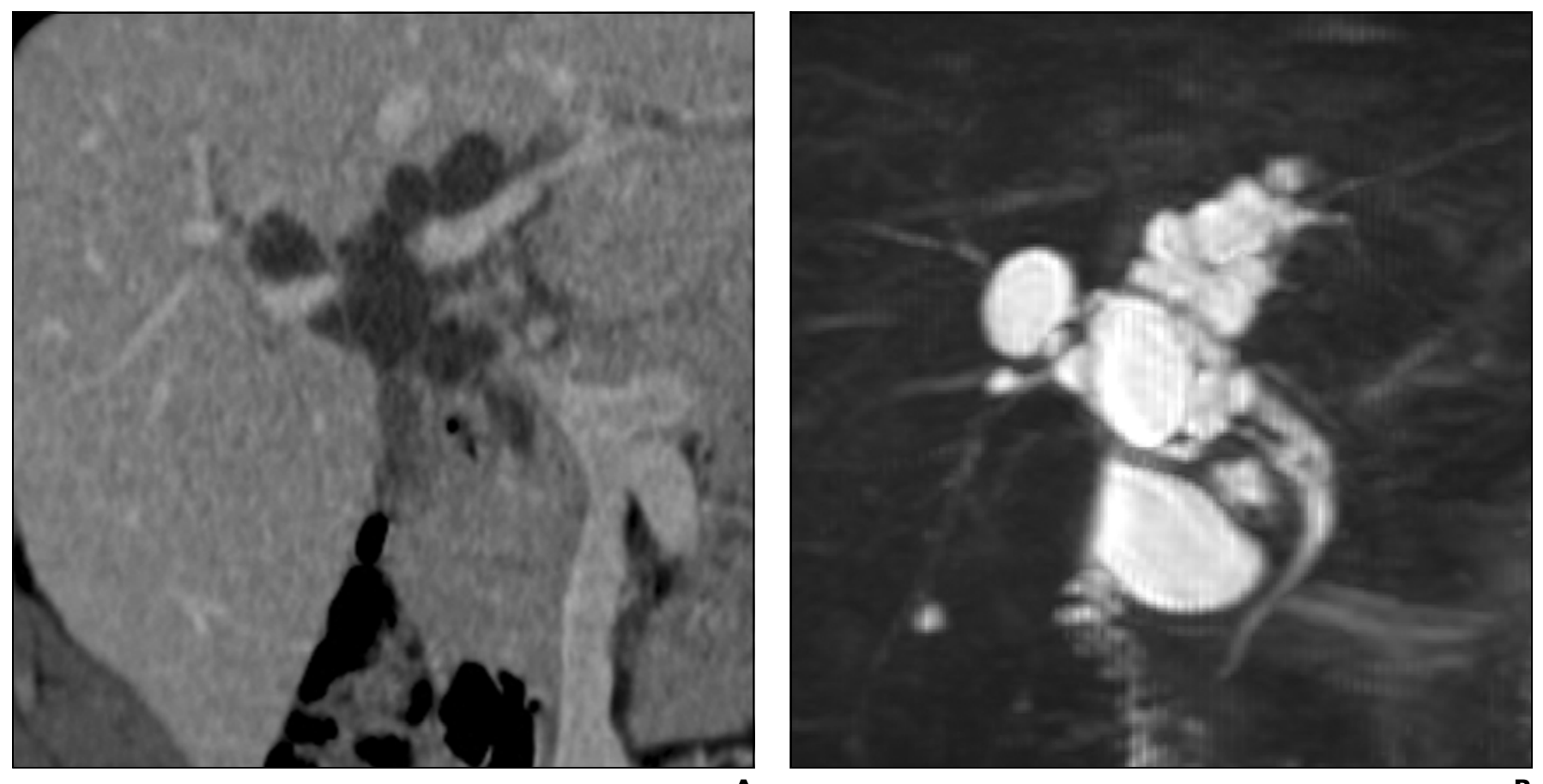

A

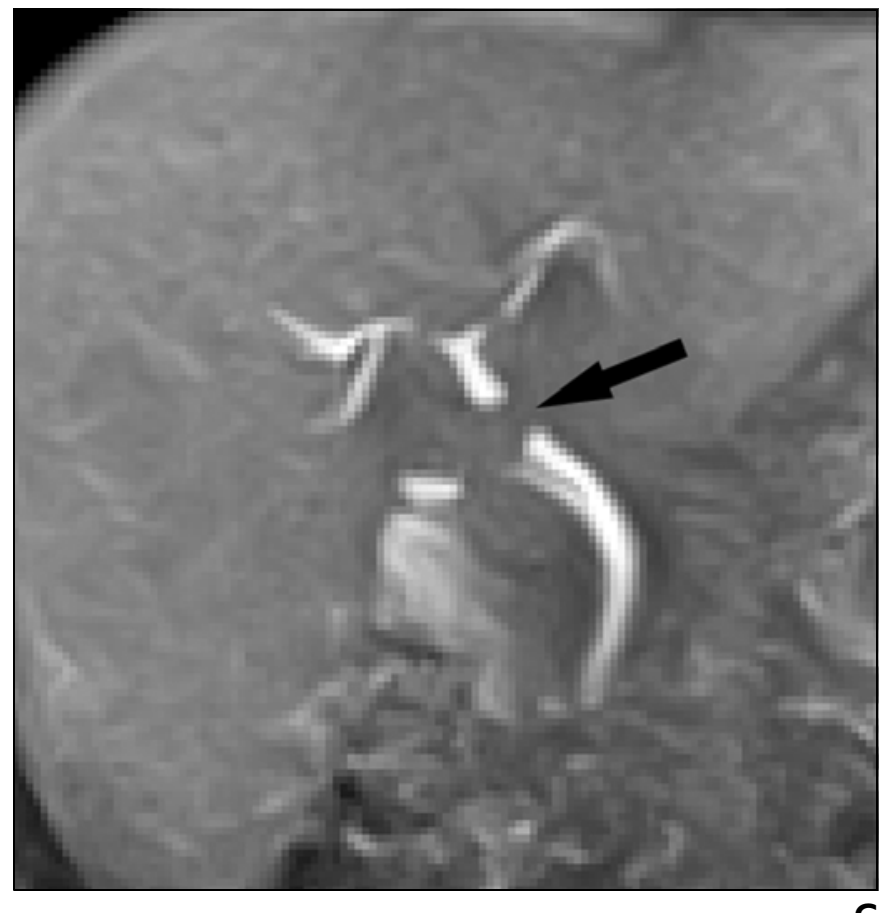

Fig. 7-22-year-old man with peribiliary cysts.

A, Minimum-intensity-projection image of MDCT shows lobulating, contoured structures with low attenuation along portal vein; this finding can be dilated bile ducts or peribiliary cystic lesions.

B, Coronal thick-slab T2-weighted MR cholangiography shows multiple round structures with high signal intensity. CT and T2-weighted MR cholangiography cannot differentiate peribiliary cysts from choledochal cysts.

C. Three-dimensional T1-weighted MR cholangiography with gadobenate dimeglumine enhancement shows normal bile duct; therefore, cystic structures are peribiliary cysts. Segmental nonvisualization of common hepatic duct was due to compression by one of the peribiliary cysts (arrow).

Regarding the assessment of hepatic masses, the unsurpassed soft-tissue contrast inherent to MRI permits assessment of the liver parenchyma using a variety of contrast mechanisms of which the T2- and contrastenhanced T1-weighted images have been shown to be the most relevant [1]. The 3D data sets of MRA were also valuable for the characterization of focal liver lesions in our study. Based on classic enhancement profiles, in the present study, two hemangiomas were diagnosed with certainty in two pa- tients, thereby obviating biopsy. In addition, chemical shift MRI has been regarded as the best diagnostic approach for detecting fatty liver [13]. For a quantitative MR assessment for fatty liver, researchers have studied using in- and opposed-phase MR sequences 


\section{Preoperative MRI of Living Liver Donors}

[28, 29]. If additional larger studies confirm the accuracy of MRI for the quantification of steatosis, MRI may render the performance of liver biopsy unnecessary for that purpose [5, 15].

Our study was clearly limited. First, our study sample was relatively small. Second, as $\mathrm{CT}$ and the surgical findings provided confirmation of our study, DSA was not performed primarily because of the reluctance of the transplant surgeons to subject otherwise healthy individuals to additional testing not deemed necessary. However, two experienced transplantation surgeons interpreted the vascular anatomy in the surgical field, and the possibility that a surgically important vascular variant was missed is very low. Last, the MR examinations were evaluated in consensus and, therefore, observer variability could not be assessed.

We conclude that a comprehensive assessment of the hepatic parenchymal; biliary ductal system; and hepatic arterial, portal, and venous systems can be accomplished with the outlined MR protocol using gadobenate dimeglumine. On the basis of our results, we conclude that MRI can potentially serve as the sole preoperative imaging test for living liver donor candidates.

\section{References}

1. Goyen M, Barkhausen J, Debatin JF, et al. Rightlobe living related liver transplantation: evaluation of a comprehensive magnetic resonance imaging protocol for assessing potential donors. Liver Transpl 2002; 8:241-250

2. Lee SY, Ko GY, Gwon DI, et al. Living donor liver transplantation: complications in donors and interventional management. Radiology 2004; 230:443-449

3. Marcos A, Fisher RA, Ham JM, et al. Selection and outcome of living donors for adult to adult right lobe transplantation. Transplantation 2000; 69:2410-2415

4. Fan ST, Lo CM, Liu CL, Yong BH, Chan JK, Ng IO. Safety of donors in live donor liver transplantation using right lobe grafts. Arch Surg 2000; 135:336-340

5. Fulcher AS, Szucs RA, Bassignani MJ, Marcos A. Right lobe living donor liver transplantation: preoperative evaluation of the donor with MR imaging. AJR 2001; 176:1483-1491
6. Lee VS, Morgan GR, Teperman LW, et al. MR imaging as the sole preoperative imaging modality for right hepatectomy: a prospective study of living adult-to-adult liver donor candidates. AJR 2001; 176:1475-1482

7. Kapoor V, Peterson MS, Baron RL, Patel S, Eghtesad B, Fung JJ. Intrahepatic biliary anatomy of living adult liver donors: correlation of mangafodipir trisodium-enhanced MR cholangiography and intraoperative cholangiography. AJR 2002; 179:1281-1286

8. Kopka L, Rodenwaldt J, Vosshenrich R, et al. Hepatic blood supply: comparison of optimized dual phase contrast-enhanced three-dimensional MR angiography and digital subtraction angiography. $R a$ diology 1999; 211:51-58

9. Morana G, Grazioli L, Testoni M, Caccia P, Procacci C. Contrast agents for hepatic magnetic resonance imaging. Top Magn Reson Imaging 2002; 13:117-150

10. Kirchin MA, Pirovano GP, Spinazzi A. Gadobenate dimeglumine (Gd-BOPTA): an overview. Invest Radiol 1998; 33:798-809

11. Earls JP, Rofsky NM, DeCorato DR, Krinsky GA, Weinreb JC. Breath-hold single-dose gadoliniumenhanced three-dimensional MR aortography: usefulness of a timing examination and MR power injector. Radiology 1996; 201:705-710

12. Michels NA. Newer anatomy of the liver and its variant blood supply and collateral circulation. Am J Surg 1966; 112:337-347

13. Mitchell DG, Kim I, Chang TS, et al. Fatty liver: chemical shift phase-difference and suppression magnetic resonance imaging techniques in animals, phantoms, and humans. Invest Radiol 1991; 26:1041-1052

14. Kreft BP, Tanimoto A, Baba Y, et al. Diagnosis of fatty liver with MR imaging. JMagn Reson Imaging 1992; 2:463-471

15. Kim SH, Lee JM, Kim JH, et al. Appropriateness of a donor liver with respect to hepatic macrosteatosis: application of artificial neural networks to US images-initial experience. Radiology 2005; 234:793-803

16. Miyazaki T, Yamashita Y, Tsuchigame T, Yamamoto H, Urata J, Takahashi M. MR cholangiopancreatography using HASTE (half-Fourier acquisition single-shot turbo spin-echo) sequences. AJR 1996; 166:1297-1303

17. Barish MA, Yucel EK, Soto JA, Chuttani R, Ferrucci JT. MR cholangiopancreatography: efficacy of three-dimensional turbo spin-echo technique. AJR 1995; 165:295-300
18. Dohke M, Watanabe Y, Okumura A, et al. Anomalies and anatomic variants of the biliary tree revealed by MR cholangiopancreatography. AJR 1999; 173:1251-1254

19. Lee VS, Rofsky NM, Morgan GR, et al. Volumetric mangafodipir trisodium-enhanced cholangiography to define intrahepatic biliary anatomy. AJR 2001; 176:906-908

20. Papanikolaou N, Prassopoulos P, Eracleous E, Maris T, Gogas C, Gourtsoyiannis N. Contrast-enhanced magnetic resonance cholangiography versus heavily T2-weighted magnetic resonance cholangiography. Invest Radiol 2001; 36:682-686

21. Wang ZJ, Yeh BM, Roberts JP, Breiman RS, Qayyam S, Coakley FV. Living donor candidates for right hepatic lobe transplantation: evaluation at CT cholangiography-initial experience. Radiology 2005; 235:899-904

22. Yeh BM, Breiman RS, Taouli B, et al. Biliary tract depiction in living potential liver donors: comparison of conventional MR, mangafodipir trisodium-enhanced excretory MR, and multi-detector row CT cholangiography-initial experience. $R a$ diology 2004; 230:645-651

23. Winter TC 3rd, Freeny PC, Nghiem HV, et al. Hepatic arterial anatomy in transplantation candidates: evaluation with three-dimensional CT arteriography. Radiology 1995; 195:363-370

24. Chan JK, Tso WK, Lo CM, et al. Preoperative evaluation of potential living donors for liver transplantation: the role of helical computed tomography-angiography. Transplant Proc 1998; 30:3197-3198

25. Eubank WB, Wherry KL, Maki JH, Sahin H, Funkhouser CP, Schmiedl UP. Preoperative evaluation of patients awaiting liver transplantation: comparison of multiphasic contrast-enhanced 3D magnetic resonance to helical computed tomography examinations. J Magn Reson Imaging 2002; 16:565-575

26. Prince MR, Arnoldus C, Frisoli JK. Nephrotoxicity of high-dose gadolinium compared with iodinated contrast. J Magn Reson Imaging 1996; 6:162-166

27. Shellock FG, Kanal E. Safety of magnetic resonance imaging contrast agents. J Magn Reson Imaging 1999; 10:477-484

28. Levenson H, Greensite F, Hoefs J, et al. Fatty infiltration of the liver: quantification with phase-contrast MR imaging at $1.5 \mathrm{~T}$ vs biopsy. AJR 1991; 156:307-312

29. Rinella ME, McCarthy R, Thakrar K, et al. Dualecho, chemical shift gradient-echo magnetic resonance imaging to quantify hepatic steatosis: implications for living liver donation. Liver Transpl 2003; 9:851-856 